\title{
The Effect of Parallel Inhomogeneity on Magnetospheric Hydromagnetic Wave Coupling
}

\author{
DAVID J. SOUTHWOOD \\ Institute of Geophysics and Planetary Physics, University of California, Los Angeles \\ Department of Physics, Imperial College of Science and Technology, London \\ MARGARET G. Kivelson \\ Institute of Geophysics and Planetary Physics and Department of Earth and Space Sciences, \\ University of California, Los Angeles
}

\begin{abstract}
In a cold homogeneous plasma, fast mode and transverse mode MHD waves can be independently excited. Inhomogeneities couple the modes and allow the excitation of field line resonances by compressional (fast mode) perturbations of the magnetosphere. Past discussions have focused on inhomogeneities in the radial direction (or its equivalent in simpler geometries), ignoring gradients of the Alfvén velocity along the unperturbed field direction. The nature of the coupling and the effectiveness of excitation are significantly modified by the presence of such gradients. One essential change is that the compressional and transverse field perturbations differ in their structure along the field. This reduces the effectiveness of the coupling between the fast (global) mode and standing (localized) field line resonances for identical harmonics of the parallel structure but allows different harmonics to couple. A fast mode wave propagating in from the outer boundary can, therefore, drive field line resonances in the region exterior to its turning point, or effective reflection point, where its amplitude has not yet decayed.
\end{abstract}

\section{INTRODUCTION}

In a cold magnetized plasma, the two low-frequency magnetohydrodynamic modes can be coupled by inhomogeneity. In magnetospheric physics the coupling gives rise to field line resonance [Chen and Hasegawa, 1974; Southwood, 1974]. Fairly simple theories of field line resonance have had considerable success in describing the large-scale spatial phase and amplitude structure of geomagnetic pulsations, smallamplitude fluctuations in the earth's field, in the ultralowfrequency band (see, for example, Lanzerotti and Southwood [1979]). The mode coupling has been regarded as important in other areas of solar terrestrial physics. In solar coronal physics, for example, similar theories have been derived to explain the heating of the corona (see, for example, Ionson [1978], Wentzel [1979], and Rae and Roberts [1981]). In a recent paper we have shown that the theoretical problem posed by the coupled hydromagnetic equations has a history extending back as far as the 1930s. The coupled equations for the hydromagnetic waves are directly paralleled by equations describing the propagation of an electromagnetic wave obliquely incident on a stratified unmagnetized plasma [Kivelson and Southwood, 1986, and references therein]. In this guise the problem has been studied in the contexts of ionospheric physics and laser fusion.

In this paper we extend the hydromagnetic box model introduced by Southwood [1974] for the study of mode coupling in an inhomogeneous cold plasma to examine the consequences of Alfvén velocity variations along the magnetic field direction. As we show, in extreme circumstances the coupling of large-scale fast mode signals to field line resonances (localized transverse modes) is substantially modified in comparison with existing models [Southwood, 1974, 1975; Kivelson and Southwood, 1985, 1986].

\section{Copyright 1986 by the American Geophysical Union.}

Paper number 5A8260.

0148-0227/86/005A-8260\$05.00

\section{MODEL}

The basic model consists of a cold plasma embedded in a uniform magnetic field $B$ in the $z$ direction [Southwood, 1974]. The plasma is of finite extent in the $z$ direction with the boundaries at $z= \pm l$ and in the $x$ direction with boundaries at $x=c$ and $x=a>c$ and periodic in $y$, as illustrated in Figure 1. Boundaries are assumed to be weakly absorptive unless we specify otherwise. Although the field is required to be uniform, we allow the density $\rho$ to vary in both $x$ and $z$. Note that in previous use of the model, the inhomogeneity has been restricted to the $x$ direction [e.g., Kivelson and Southwood, 1985]. The central interest of this paper is in the effect of the inhomogeneity in $z$ (along the background field).

From Faraday's law, the momentum equation, and frozenin fields [e.g., Southwood and Hughes, 1983], the governing equations for small-amplitude hydromagnetic waves in the model plasma may be obtained in the form

$$
\begin{gathered}
\left(\omega^{2} \mu_{0} \rho / B^{2}+\partial^{2} / \partial z^{2}\right) \xi_{x}=B^{-1} \partial b_{z} / \partial x \\
\left(\omega^{2} \mu_{0} \rho / B^{2}+\partial^{2} / \partial z^{2}\right) \xi_{y}=i \lambda B^{-1} b_{z} \\
b_{z} / B=-\partial \xi_{x} / \partial x-i \lambda \xi_{y}
\end{gathered}
$$

where $\xi_{x}$ and $\xi_{y}$ are $x$ and $y$ components of the plasma displacement vector and $b_{z}$ is the compressional field perturbation. Here $\lambda$ is the wave number in $y$, and $\omega$ is the angular frequency. The wave field components have been assumed to vary as exp $(i \lambda y-i \omega t)$. If we eliminate $\xi_{x}$ and $\xi_{y}$ from (1)-(3), we find a lourth-order partial differential equation for $b_{z}$ :

$$
\left(K^{2}+\frac{\partial^{2}}{\partial z^{2}}\right)\left(\frac{\partial K^{2}}{\partial x}\right)^{-1}\left(K^{2}+\frac{\partial^{2}}{\partial x^{2}}+\frac{\partial^{2}}{\partial z^{2}}-\lambda^{2}\right) b_{z}=\frac{\partial b_{z}}{\partial x}
$$

where $K^{2}=\omega^{2} \mu_{0} \rho / B^{2}=\omega^{2} / A^{2}$ where $A(x, z)$ is the local Allvén speed.

\section{THE UNCOUPLED MODES}

The presence of two differential operators on the left-hand side of (4) indicates that it describes two coupled wave modes. 


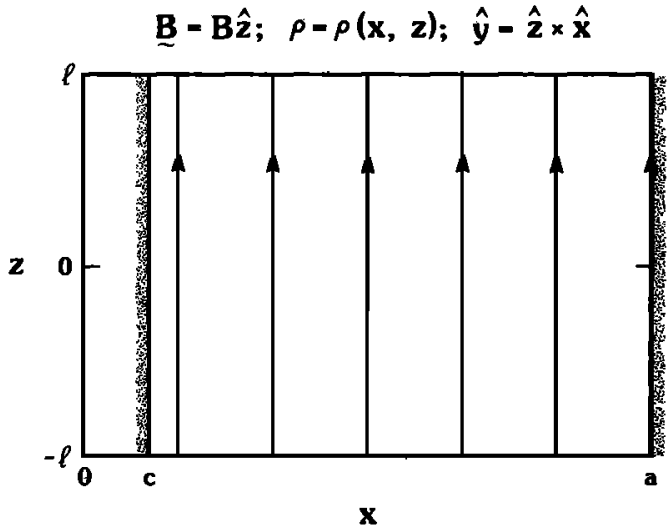

Fig. 1. Schematic of the box model coordinate system.

Note that the coupling appears to be introduced by the dependence of $K^{2}$ on the $x$ coordinate. We can show this explicitly by considering the case where there is no dependence on $x$ in the unperturbed system.

By taking the $x$ derivative of (1), multiplying (2) by $i \lambda$, adding the resulting equations, and substituting for $b_{z}$ from (3) one finds the following equation for $b_{z}$ :

$$
\left(\frac{\omega^{2}}{A^{2}}+\frac{\partial^{2}}{\partial x^{2}}+\frac{\partial^{2}}{\partial z^{2}}-\lambda^{2}\right) b_{z}=0
$$

Similarly, one may derive an equation governing the parallel vorticity $\Omega$ by eliminating $b_{z}$ from (1) and (2) and by substituting

$$
\Omega=-i \omega\left(\frac{\partial \xi_{y}}{\partial x}-i \lambda \xi_{x}\right)
$$

One finds that the parallel vorticity is governed by

$$
\left(\frac{\omega^{2}}{A^{2}}+\frac{\partial^{2}}{\partial z^{2}}\right) \Omega=0
$$

The existence of the two independent differential equations (5) and (7) shows that there are two uncoupled hydromagnetic modes when the only field inhomogeneity is in the $z$ direction. As in the uniform case these are the fast (equation (5)) and transverse (equation (7)) modes. The fast (compressional) mode is characterized by the field component $b z$, and the transverse mode is characterized by the parallel vorticity $\Omega$. As discussed for the uniform case by Dungey [1968], for instance, the fast mode can propagate energy in any direction relative to the field subject to the form of the Alfvén velocity distribution in $z$. Energy in the transverse mode is strictly field guided as is shown by the presence of derivatives only in $z$ in (7).

\section{Qualitative Features of The Solutions}

Let us now return to the coupled wave case. In some circumstances, we can eliminate the $z$ derivatives in (4). All earlier work has been done for cases when one may do so. Assume for the moment that $K^{2}$ is a function of $x$ alone and that the boundary conditions at $z= \pm l$ are independent of $x$. For this, Kivelson and Southwood $[1985,1986]$ derive the second-order ordinary differential equation

$$
\frac{d^{2} b_{z}}{d x^{2}}-\frac{d K^{2} / d x}{K^{2}(x)-k^{2}} \frac{d b_{z}}{d x}+\left[K^{2}(x)-k^{2}-\lambda^{2}\right] b_{z}=0
$$

where the explicit dependence of the wave field on $z$ ( $e^{i k z}$ $\pm e^{-i k z}$ ) has been suppressed for now.
Let us examine the derivation of (8). In order to satisfy the equation, the wave field must also satisfy a one-dimensional eigenvalue equation with the form

$$
\left(\frac{\partial^{2}}{\partial z^{2}}+k^{2}\right) \Omega_{k}(x, z)=0
$$

at each value of $x$. The field $b_{z}$ is thus proportional to $\Omega_{k}(x, z)$, the eigenfunction. The parameter $k$, introduced as the root of the eigenvalue, is the parallel wave number.

Note that the function $\Omega_{k}(x, z)$ is not an eigenfunction of the decoupled transverse mode, but it is closely related to one. The transverse mode dispersion relation is satisfied only where

$$
K^{2}(x)=\dot{k}^{2}
$$

Condition (10) holds only for particular values of $x$, i.e., at particular magnetic shells or field line resonances. The variation of an idealized decoupled transverse signal thus exhibits singular behavior; it has a delta function behavior in the $x$ coordinate direction while its variation along the field is given by an eigenfunction of (9), i.e., the transverse mode eigenfunction varies as $\Omega_{k}(x, z) \delta\left(x-x_{0}\right)$ where $x_{0}$ is a solution of (10).

Now when (8) is valid, the coupling between transverse and fast modes is represented by the occurrence of a singularity in the diflerential equation at any point where (10) holds.

Physically, the singularity implies the occurrence of energy absorption at the resonance. Energy is fed irreversibly into the resonance region at a rate that is independent of the local dissipation rate. As a consequence, the global part of the signal described by (8) is damped [Kivelson and Southwood, $1985]$.

In deriving (8) and eliminating the $z$ derivatives that occur in (4), we assumed that the field satisfying (8) also satisfies the differential equation (9). The latter requirement implies that the $z$ variation of the fast mode matches the amplitude variation of an isolated transverse mode signal with the same frequency. Note that it does so at all points of the resonant magnetic shell. For this to be assured, the solution must be separable in $x$ and $z$. In very simple geometries, separable solutions are obtainable, but there is no a priori guarantee of their existence, as we show below. Normally, when $K^{2}$ is a function of both $x$ and $z$, separation of the fast mode solution is not possible, and the coupling process is more subtle.

Let us consider now what happens if $K^{2}$ is regarded as a function of $x$ and $z$. The eigenvalue equation for the transverse mode operator, the operator in the first parentheses of (4), may then be written in a modified form in which the $x, z$ variation of $K^{2}$ is contained in the Alfvén velocity, $A(x, z)$,

$$
\left[A^{2}(x, z) \frac{\partial^{2}}{\partial z^{2}}+\omega_{T}^{2}(x)\right] \Omega_{T}(x, z)=0
$$

In (11), $\Omega_{T}(x, z)$ is the eigenfunction corresponding to the eigenfrequency $\omega_{T}(x)$. The allowed values of $\omega_{T}(x)$ are quantized by the $z$ boundaries as were those of $k^{2}$ before. The possible $x$ dependence of the eigenvalue is allowable because the differential part of the operator depends only on $z$ (one consequence of the field guidance of the transverse mode).

As in the previous case, the transverse mode equation can be satisfied only on isolated magnetic shells, namely at values of $x$ such that

$$
\omega^{2}=\omega_{T}^{2}(x)
$$

Equation (12) gives the resonant magnetic shells. As before, an eigenfunction for the idealized decoupled transverse mode will 
exist only on a resonant shell and thus has a delta function variation in the $x$ direction; i.e., the eigenfunction is of the form $\Omega_{T}(x, z) \delta\left(x-x_{0}\right)$ where $x_{0}$ is the resonant shell.

The uncoupled fast mode equation, derived from the operator in the second parentheses in (4), can be written

$$
\left[A^{2}(x, z)\left(\frac{\partial^{2}}{\partial x^{2}}+\frac{\partial^{2}}{\partial z^{2}}-\lambda^{2}\right)+\omega_{F}^{2}\right] b_{z}=0
$$

(cf. also equation (8)).

The eigenvalues of (13), $\omega_{F}$, are constant and are quantized by the combination of $x$ and $z$ boundary conditions. The eigenfunctions will have a well-defined variation in $x$ and $z$, but note that the form of the dependence on $z$ on any particular shell differs from that of the transverse mode solution derived from (11). To demonstrate this point, we substitute a transverse mode eigenfunction into the eigenvalue equation (13). One finds

$$
\left[A^{2}(x, z)\left(\frac{\partial^{2}}{\partial x^{2}}-\lambda^{2}\right)-\omega_{T}{ }^{2}(x)+\omega_{F}{ }^{2}\right] \Omega_{T}(x, z)=0
$$

Note how the substitution has not removed all $z$ dependence from the operator. One concludes that the dependence on $z$ of an eigenfunction for the fast mode equation will not generally match that of a transverse mode eigenfunction along any particular field line. The consequences of this are best pursued by first looking at a simpler density model.

\section{SOLUTION FOR DENSITY INDEPENDENT OF $x$}

Let us now return to the problem posed by the box model with density variation only along the field in the $z$ direction. In this section we shall assume that all boundaries of the box are perfectly reflecting. The coupling of modes is completely removed if $K^{2}(x, z)$ is independent of $x$ as we discussed earlier.

The transverse mode is governed by (10). Thus its eigenfunctions $\Omega_{T}$ must satisfy the following equation:

$$
\left[A^{2}(z) \frac{\partial^{2}}{\partial z^{2}}+\omega_{T}^{2}\right] \Omega_{T}(x, z)=0
$$

The eigenvalues of $(15), \omega_{T}$, are constant, as the system contains no $x$ dependence; the lack of specific reference to $x$ in the transverse operator also implies that the variation with $x$ of the transverse mode is arbitrary rather than singular as in the previous case.

The governing equation for the fast mode eigenfunctions is (8) and can be written in the form

$$
\left[A^{2}(z)\left(\frac{\partial^{2}}{\partial x^{2}}+\frac{\partial^{2}}{\partial z^{2}}-\lambda^{2}\right)+\omega_{F}^{2}\right] b_{z}=0
$$

It is possible to find separable solutions analogous to those found above for both (15) and (16). For the fast mode we may assume a sinusoidal variation in $x$ characterized by a wave number $v$. We thus obtain an equation in $z$ alone,

$$
\left[A^{2}(z)\left(\frac{\partial^{2}}{\partial z^{2}}-v^{2}-\lambda^{2}\right)+\omega_{F}^{2}\right] b_{z}=0
$$

and again the dependence on the suppressed variables is understood.

The solutions to (17) are analogous in structure to solutions to (8), for they are spatially oscillatory in limited regions and damp (or grow monotonically) elsewhere. The turning point, where the nature of the solutions changes, is given in each case by the locus of points for which the coefficient of the term not containing any derivatives is zero. In the latter case, the turning points of the equation are given by particular values of $x$. In the former case, the condition yields a set of $z$ values such that

$$
\omega^{2} / A^{2}\left(z_{v}\right)=v^{2}+\lambda^{2}
$$

If we suppose that the dense plasma is concentrated near $z=0$, in rough analogy to the concentration of plasma near the equator under some magnetospheric conditions (as, for example, near Io in the Jovian magnetosphere), then the solutions are oscillatory for $|z|<z_{v \lambda}$ and damp for $|z|>z_{v \lambda}$.

Let us assume a particular density model. Suppose that near the center of the field lines, $|z|<z_{0}, \rho=\rho_{0}$ and for $|z|>z_{0}$, $\rho=\rho_{1}$ and that the corresponding Alfvén speed for each region is $A_{0}$ and $A_{1}$, respectively. For given $v, \lambda$, the lowestfrequency fast mode solution is that with the slowest variation along $z$. Let us take $b_{z}$ to be zero at the boundaries. As the system is symmetric about $z=0$, solutions are either even or odd functions of $z$. An even solution would take the form

$$
\begin{array}{ll}
b_{z}=C \sinh \left[\kappa_{1}\left(l-z_{0}\right)\right] \cos K_{0} z & |z| \leq z_{0} \\
b_{z}=C \sinh \left[\kappa_{1}(l-|z|)\right] \cos K_{0} z_{0} & z_{0}<|z|
\end{array}
$$

where $C$ is an arbitrary amplitude constant. An odd solution is antisymmetric about $z=0$ and for positive $z$ varies as

$$
\begin{array}{ll}
b_{z}=C \sinh \left[\kappa_{1}\left(l-z_{0}\right)\right] \sin K_{0} z & 0 \leq z \leq z_{0} \\
b_{z}=C \sinh \left[\kappa_{1}(l-z)\right] \sin K_{0} z_{0} & z_{0}<z
\end{array}
$$

In both cases

$$
K_{0}{ }^{2}=\frac{\omega_{F}{ }^{2}}{A_{0}{ }^{2}}-\lambda^{2}-v^{2} \quad \kappa_{1}{ }^{2}=\lambda^{2}+v^{2}-\frac{\omega_{F}{ }^{2}}{{A_{1}}^{2}}
$$

The eigenvalues are determined by the roots of the following two equations:

$$
\begin{aligned}
& K_{0} \tan K_{0} z_{0}=\kappa_{1} \operatorname{coth}\left[\kappa_{1}\left(l-z_{0}\right)\right] \\
& K_{0} \cot K_{0} z_{0}=-\kappa_{1} \operatorname{coth}\left[\kappa_{1}\left(l-z_{0}\right)\right]
\end{aligned}
$$

All eigenfrequencies derived from (22) and (23) depend on $v$ and $\lambda$, but that is not important to our discussion here. The structure parallel to the field is important, and we should briefly discuss it, assuming that $v$ and $\lambda$ are fixed. Equation (22) always has the smallest root, so the lowest-frequency eigenmode is symmetric about $z=0$, i.e., is an even function. A sketch of the $z$ variation of $b_{z}$ is given in Figure 2. The next higher frequency corresponds to an eigenmode of (23), i.e., antisymmetric about $z=0$, and successively higher-frequency

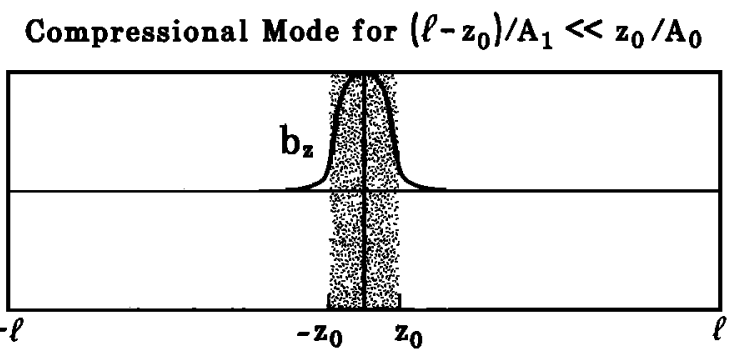

Fig. 2. The parallel magnetic perturbation for the fundamental harmonic of the structure along the background magnetic field in the box model of an inhomogeneous plasma with density $\rho_{0}$ for $|z|<z_{0}$ and density $\rho_{1} \ll \rho_{0}$ for $|z|>z_{0}$. The perturbation field is plotted versus distance along the field line between boundaries at $\pm l$. The case illustrated corresponds to the limit of $\kappa_{1}\left(l-z_{0}\right) \gg 1$ discussed in the text. 
Transverse Mode for $\left(\ell-z_{0}\right) / A_{1} \gg z_{0} / A_{0}$
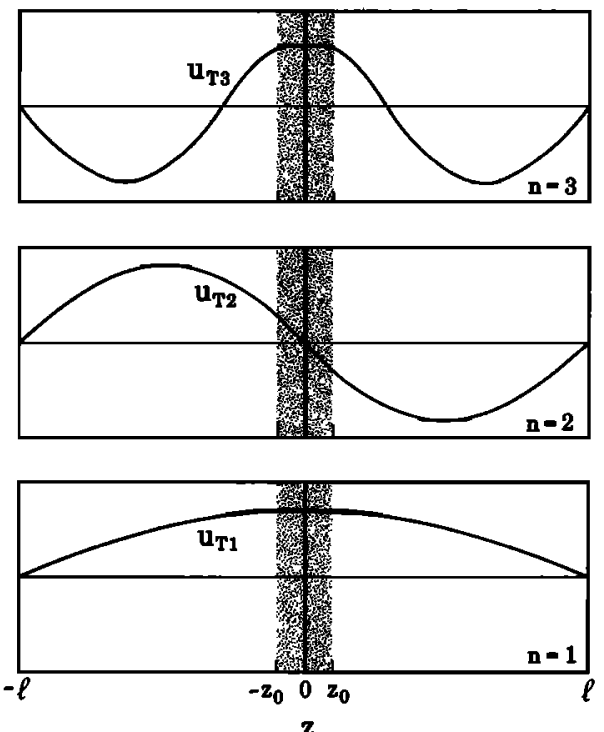

Fig. 3. The plasma displacement for the first three harmonics of the structure along the background magnetic field in the box model with density distribution as in Figure 2 . The case illustrated corresponds to the limit of $\left(l_{0}-z\right) / A_{1} \gg z_{0} / A_{0}$.

solutions continue to alternate between even and odd symmetry. At some point the frequency will be high enough that the variation in the low-density region will become sinusoidal instead of hyperbolic.

In general, (22) and (23) are transcendental, but there is one interesting limit. If $\kappa_{1}\left(l-z_{0}\right) \gg 1$, the wave amplitude is essentially confined to the region $|z|<z_{0}$. For fixed $v$ and $\lambda$ the lowest eigenfrequency is given by

$$
K_{0} z_{0} \lesssim \pi / 2
$$

that is,

$$
\omega_{F}^{2} \lesssim A_{0}^{2}\left(\frac{\pi^{2}}{4 z_{0}^{2}}+v^{2}+\lambda^{2}\right)
$$

and the inequality is weak provided $z_{0} / l$ is of order unity.

Now let us return to consider the transverse mode described by (15). Let us assume that the wave field in the equation is a component of the plasma displacement and that it is zero at the boundaries. The solutions of (15) can now be found and can also be classified by their odd or even symmetry with respect to $z=0$. A solution with even symmetry takes the form

$$
\begin{array}{ll}
\mathbf{\Omega}_{T}=C \sin \left[\omega\left(l-z_{0}\right) / A_{1}\right] \cos \left(\omega z / A_{0}\right) & |z| \leq z_{0} \\
\mathbf{\Omega}_{T}=C \sin \left[\omega(l-|z|) / A_{1}\right] \cos \left(\omega z_{0} / A_{0}\right) & z_{0}<|z|
\end{array}
$$

The odd mode is antisymmetric with respect to $z=0$ and for $z>0$ takes the form

$$
\begin{array}{ll}
\Omega_{T}=C \sin \left[\omega\left(l-z_{0}\right) / A_{1}\right] \sin \left(\omega z / A_{0}\right) & 0 \leq z \leq z_{0} \\
\Omega_{T}=C \sin \left[\omega(l-z) / A_{1}\right] \sin \left(\omega z_{0} / A_{0}\right) & z_{0}<z
\end{array}
$$

The eigenfrequencies are given by the solutions of the following two equations:

$$
\begin{aligned}
& A_{0}{ }^{-1} \tan \left(\omega_{T} z_{0} / A_{0}\right)=A_{1}{ }^{-1} \cot \left[\omega_{T}\left(l-z_{0}\right) / A_{1}\right] \\
& A_{0}{ }^{-1} \cot \left(\omega_{T} z_{0} / A_{0}\right)=-A_{1}{ }^{-1} \cot \left[\omega_{T}\left(l-z_{0}\right) / A_{1}\right]
\end{aligned}
$$

Equations (27) and (28) correspond to even (symmetric in z) modes and odd (antisymmetric in z), respectively. As with the fast mode, the symmetric case (27) yields the lowest eigenfrequency, i.e., characterizes the lundamental. Unlike the fast mode case, the eigenfrequencies are not affected by the signal variation across the field.

Two extreme cases merit consideration. The limits are $\left(l-z_{0}\right) / A_{1}$ much greater than or much less than $z_{0} / A_{0}$ and correspond to the Alfven travel time being much larger inside or outside the high-density region. Sketches of the eigenfunctions are shown in Figures 3 and 4.

Figure 3 shows what occurs if $\left(l-z_{0}\right) / A_{1} \gg z_{0} / A_{0}$. The amplitude of the displacement changes little in the central region, and the frequencies of the lowest harmonic structures along the field are determined by the field line length $2 l$. In the opposite extreme, $\left(l-z_{0}\right) / A_{1} \ll z_{0} / A_{0}$, the scale of the highdensity region, $z_{0}$, determines the eigenfrequency structure, and the mode is more effectively confined to the high-density part of the flux tube. In this case, sketches are shown of the transverse field component as well as the plasma displacement.

\section{Coupled Mode Solutions}

Let us now return to consider (4). We shall assume that there are strong gradients in density parallel to the field at some point on each flux tube. Later when we specialize, we will return to a model similar to that in the previous section. The parallel density gradients preclude us from using the approach outlined for (8).

Let $G\left(x, z ; z^{\prime}\right)$ be the Green's function for the transverse mode operator in (11). Because the operator is self-adjoint, its eigenfunctions form a complete set for functions satisfying the boundary conditions imposed at the $z$ boundaries. As a result the Green's function can be expressed formally in terms of a series of the normalized eigenfunctions, $\Omega_{T i}(x, z)$, with eigenvalues $\omega_{T i}$ as

$$
G\left(x, z ; z^{\prime}\right)=\sum_{i} \frac{\Omega_{T i}(x, z) \Omega_{T i}\left(x, z^{\prime}\right)}{\omega^{2}-\omega_{T i}{ }^{2}(x)}
$$

Transverse Mode for $\left(\ell-z_{0}\right) / A_{1} \ll z_{0} / A_{0}$
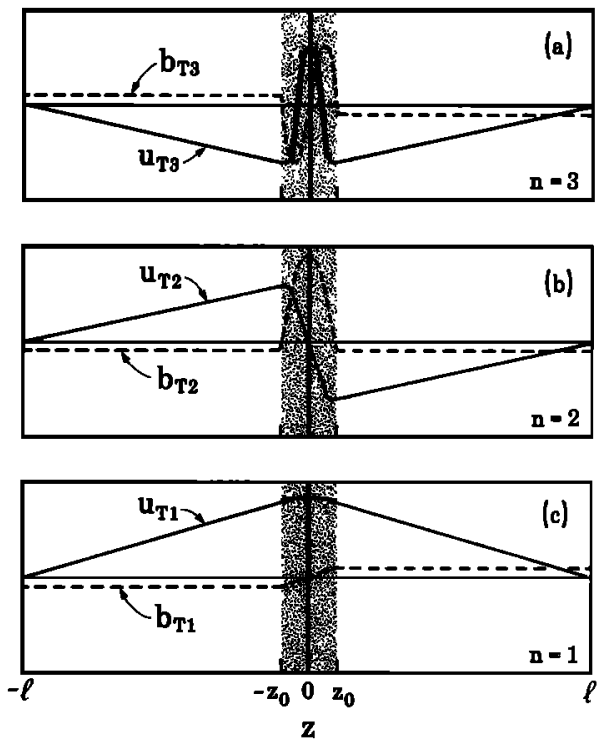

Fig. 4. The plasma displacement (solid curves) and the transverse magnetic perturbation field (dashed curves) for the first three harmonics of the transverse mode as in Figure 3. The case illustrated corresponds to the limit of $\left(l-z_{0}\right) / A_{1} \ll z_{0} / A_{0}$. 
Replacing $z$ by $z^{\prime}$ in (4), we multiply it by $G\left(x, z ; z^{\prime}\right)$ and integrate the resulting expression with respect to $z^{\prime}$ over the length of the flux tubes. We thus obtain the following formal inversion of the transverse operator in (4);

$$
\begin{aligned}
& A^{-2}\left[K^{2}(x, z)+\frac{\partial^{2}}{\partial x^{2}}+\frac{\partial^{2}}{\partial z^{2}}-\lambda^{2}\right] b_{z} \\
& =\frac{\partial K^{2}}{\partial x} \int_{-1}^{l} d z^{\prime} G\left(x, z ; z^{\prime}\right) \frac{\partial b_{z}\left(x, z^{\prime}\right)}{\partial x}+\text { boundary terms }
\end{aligned}
$$

Now consider the term involving the Green's function. It can be written

$$
\begin{aligned}
\frac{\partial K^{2}}{\partial x} \sum_{i} \frac{\Omega_{T i}(x, z)}{\omega^{2}-\omega_{T i}{ }^{2}(x)} & \int_{-l}^{l} d z^{\prime} \Omega_{T i}\left(x, z^{\prime}\right) \frac{\partial b_{z}\left(x, z^{\prime}\right)}{\partial x} \\
& \equiv \frac{\partial K^{2}}{\partial x} \sum_{i} \frac{\Omega_{T i}(x, z)}{\omega^{2}-\omega_{T i}{ }^{2}(x)}\left\langle\Omega_{T i} \mid \frac{\partial b_{z}}{\partial x}\right\rangle
\end{aligned}
$$

Although the summation is over all eigenvalues, near any field line such that $\omega^{2}=\omega_{T n}{ }^{2}(x)$, the $n$th term will dominate unless the bracket, $\left\langle\Omega_{T n} \mid \partial b_{z} / \partial x\right\rangle$, vanishes.

Formally, one should be able to write

$$
b_{z}=\sum_{i} \Omega_{T i}(x, z)\left\langle\Omega_{T i} \mid b_{z}\right\rangle
$$

Near the resonant shell, $\mathbf{\Omega}_{T_{n}}(x, z)$ will vary smoothly with $x$. We thus can approximate it by its value on the resonant shell. By extracting from (30) only those terms varying as $\Omega_{T n}(x, z)$, we obtain a differential equation for the $n$th coeflicient in the series (32):

$$
\begin{aligned}
{\left[\frac{\partial^{2}}{\partial x^{2}}+\frac{\omega^{2}-\omega_{T n}{ }^{2}(x)}{A^{2}(x, z)}-\right.} & \left.\lambda^{2}\right]\left\langle\Omega_{T n} \mid b_{z}\right\rangle \\
& =A^{2} \frac{\partial}{\partial x}\left(\frac{\omega^{2}}{A^{2}}\right) \frac{(\partial / \partial x)\left\langle\Omega_{T_{n}} \mid b_{z}\right\rangle}{\omega^{2}-\omega_{T_{n}}{ }^{2}(x)}
\end{aligned}
$$

where the small boundary terms have been omitted. Near the resonance the second term on the left side of the equation may be dropped. If, in addition, $A^{2}$ is a separable function of $x$ and $z$, the $z$ dependence disappears, and the equation has the same form as (4) near its resonant point, $K^{2}=k^{2}$.

The solutions of (4) near the singularity have been discussed by several authors [Kivelson and Southwood, 1986, and references therein]. The compressional field perturbation $b_{z}$ remains finite at the singular point, but it is nonanalytic, and its second derivative is discontinuous.

Let us now return to (2). Near the resonance, $b_{z}$ is finite, and thus the amplitude of the plasma displacement, $\xi_{y n}$, of the $n$th harmonic is approximately given by the formal solution

$$
\xi_{y n}=\frac{B}{\mu_{0}} i \lambda \frac{b_{z n}\left(x_{\omega n}\right) \Omega_{T n}\left(x_{\omega n}, z\right)}{\left[\omega^{2}-\omega_{T n}{ }^{2}(x)\right] \rho\left(x_{\omega n}\right)}
$$

with

$$
b_{z n}=\left\langle\Omega_{T n}\left(x_{\omega n}, z\right) \mid b_{z}\left(x_{\omega n}, z\right)\right\rangle
$$

where $x_{\omega n}$ satisfies $\omega^{2}=\omega_{T n}{ }^{2}\left(x_{\omega n}\right)$. The displacement $\xi_{x n}$ will vary logarithmically with $x$ near the resonance [Kivelson and Southwood, 1986], and a formal solution can be found by using (34) in (3) and noting that in the latter equation the term in $b_{z}$ is negligible near the singularity.

There is an overt similarity between the form of the solutions of (8) and those given in (33) and (34). Note, however, that the solutions given in (33) and (34) are approximate solu- tions to the fully coupled equation (4). They represent the dominant resonant terms near the shell where the compressional global mode frequency matches one of the local eigenfrequencies of the transverse mode. In different regions, different harmonics of the transverse mode can match the global mode frequency. A given global mode with a fixed frequency may match the fundamental transverse mode (field line resonance) frequency in the highest-density regions and then match successive harmonics of that frequency as one moves to larger values of $x$. Let us now explore the consequences of such coupling further.

\section{Discussion OF THE COUPLED SOlution}

The formal approach of the preceding section should not be allowed to hide some simple conclusions. An important new effect has emerged. In the case where there is significant inhomogeneity along the magnetic field, a fast mode signal at a particular frequency with a given structure along the magnetic field can generally excite multiple transverse mode field line resonances. All will have a common frequency $\omega$ but will correspond to different harmonics of structure parallel to the field. In a system in which the density varies monotonically with $x$, the fast mode signal will excite the different harmonics in well-separated localized regions. However, we should now recognize that it will not excite all harmonics in a system that is symmetric about the field line equator. The symmetry in $z$ of the plasma displacement of the localized mode must be the same as that of the compressional component $b_{z}$ (cf. equations (2) and (3)).

Let us now consider a situation akin to that discussed earlier in which there is a high-density region concentrated around $z=0$. The $b_{z}$ structure we expect is shown in Figure 2, with the amplitude largest in the high-density region. Its frequency is given by (24). The transverse mode structure depends on the ratio of Alfvén travel times for the high- and low-density regions. In the case where the Alfven travel time is largest in the low-density regions, the lowest Alfven wave harmonics have frequencies controlled by the field line length, $2 l$. Now the lowest fast mode frequency is larger than $\pi A_{0} / 2 z_{0}$ (equation (24)). Unless $z_{0}$ is of the order of $l$, we can conclude that the frequency matching will be possible only for high transverse mode harmonics. One does not expect coupling of high harmonics to be very efficient in view of the difference in functional form of such harmonics to the fundamental structure for $b_{z}$ (Figure 2).

In contrast, in the case where the Alfven travel time is larger in the high-density region near $z=0$, the frequency of the fundamental and first few harmonics is governed by $z_{0}$, and if we assume that for the fast mode $v^{2}, \lambda^{2}$ are of the order of $\left(\pi A_{0} / 2 z_{0}\right)^{2}$, then it is not unreasonable to expect frequency matching with the lower harmonics of the transverse mode at some point in the system. In the case of (8), only the fundamental transverse mode resonance can be excited by the fundamental compressional mode, and this occurs on a shell where the fast mode is nonpropagating, i.e., field line resonance occurs beyond the turning point. The fast mode, if it is propagating, inevitably has a frequency higher than the local fundamental transverse mode frequency. We should thus inquire if one could expect efficient coupling between the fast mode fundamental and, say, second or third harmonics of the transverse mode which could occur in the region where the fast mode propagates. Such coupling could not occur in the case where (8) holds. 
A measure of the strength of the coupling is the size of the term in brackets, $b_{z n}$ (cf. equation (35)).

Comparison of Figures 2 and 4 shows that coupling to the $n=2$ mode is impossible because of the opposite symmetry but coupling to the $n=3$ mode could be quite efficient.

\section{Conclusions}

In this paper we have investigated the effect on the field line resonance problem of gradients in Alfvén speed parallel to the background field. Even in the hydromagnetic box model, the general governing equation is a partial differential equation of fourth order. By dint of examining special density distributions, we have shown that the field line resonance phenomenon is still expected to occur as in the case where the gradients are solely across the field. One essential change is that the compressional and transverse field perturbations differ in their structure along the field. This reduces the effectiveness of the coupling between the fast (global) mode and standing (localized) field line resonances for identical harmonics of the parallel structure but allows different harmonics to couple. A fast mode wave propagating in from the outer boundary can, therefore, drive field line resonances in the region exterior to its turning point, or effective reflection point, where its amplitude has not yet decayed.

We have particularly emphasized the effectiveness of coupling of the fast mode to the third harmonic Alfven mode in a model where a high-density region is confined near the center of the field line. This case is potentially applicable to hydromagnetic wave structures in the Jovian magnetosphere where the centrifugal distortion of the density distribution creates a high-density zone about the field line equator.

The relevance of the extreme density configurations we have invoked here to the terrestrial magnetosphere is less apparent. However, the principles we have outlined surely apply to the inhomogeneous terrestrial system and may be useful conceptually in attacking the full coupled-mode problem in a dipole background field. Many dayside compressional waves in the terrestrial magnetosphere appear consistent with strong localization near the magnetic equator [e.g., Barfield and Lin, 1983; Nagano and Araki, 1983; Engebretson et al., 1986], as we would expect if the low-latitude plasma density is high. Further work is needed to test the applicability of our conclusions to the observed wave properties, but it is already clear that it will be important to organize the wave data not only by magnetic shell values but also by magnetic latitude.
Acknowledgments. The authors gratefully acknowledge a useful discussion with V. A. Pilipenko which sparked their interest in this problem. The work was supported by the Division of Atmospheric Sciences of the National Science Foundation under grant ATM 8300523. This is UCLA Institute of Geophysics and Planetary Physics publication 2697.

The Editor thanks $\mathrm{L}$. Chen and another referee for their assistance In evaluating this paper.

\section{REFERENCES}

Barfield, J. N., and C. S. Lin, Remote determination of the outer radial limit of stormtime Pc 5 wave occurrence using geosynchronous satellites, Geophys. Res. Lett., 10, 671, 1983.

Chen, L., and A. Hasegawa, A theory of long period magnetic pulsations, 1, Steady state excitation of field line resonance, J. Geophys. Res., 79, 1024, 1974.

Dungey, J. W., Hydromagnetic waves, in Physics of Geomagnetic Phenomena, edited by W. H. Campbell and S. Matsushita, p. 913, Academic, Orlando, Fla., 1968.

Engebretson, M. J., L. J. Cahill, Jr., J. H. Waite, Jr., D. L. Gallagher, M. O. Chandler, M. Sugiura, and D. Weimer, Wave and plasma observations during a compressional Pc 5 wave event August 10, 1982, J. Geophys. Res., 9I, 6884, 1986.

Ionson, J. A., Resonant absorption of Alfvenic surface waves and heating of coronal loops, Astrophys. J., 226, 650, 1978.

Kivelson, M. G., and D. J. Southwood, Resonant ULF waves: A new interpretation, Geophys. Res. Lett., 12, 49, 1985.

Kivelson, M. G., and D. J. Southwood, Coupling of global magnetospheric MHD eigenmodes to field line resonances, $J$. Geophys. Res., 91, 4345, 1986.

Lanzerotti, L. J., and D. J. Southwood, Hydromagnetic waves, in Solar System Plasma Physics, vol. III, edited by E. N. Parker, C. F. Kennel, and L. J. Lanzerotti, p. 109, North-Holland, Amsterdam, 1979.

Nagano, H., and T. Araki, Long-duration Pc 5 pulsations observed by geostationary satellites, Geophys. Res. Lett., 10, 908, 1983.

Rae, I. C., and B. Roberts, Surface waves and the heating of the corona, Geophys. Astrophys. Fluid Dyn., 18, 197, 1981.

Southwood, D. J., Some features of field line resonances in the magnetosphere, Planet. Space Sci., 22, 483, 1974.

Southwood, D. J., Comments on field line resonances and micropulsations, Geophys. J. R. Astron. Soc., 4I, 425, 1975.

Southwood, D. J., and W. J. Hughes, Theory of hydromagnetic waves in the magnetosphere, Space Sci. Rev., 35, 301, 1983.

Wentzel, D. G., The dissipation of hydromagnetıc surface waves, Astrophys. J., 233, 756, 1979.

M. G. Kivelson, Department of Earth and Space Sciences, 3805 Geology Building, University of California, Los Angeles, CA 90024.

D. J. Southwood, Department of Physics, Imperial College of Science and Technology, London SW7 2BZ, England.

(Received October 10, 1985; revised March 11, 1986; accepted March 11, 1986.) 